Collection SFN 10 (2010) 137-155

(C) Owned by the authors, published by EDP Sciences, 2010

DOI: $10.1051 / \mathrm{sfn} / 2010002$

\title{
Des propriétés électroniques aux excitations magnétiques
}

\author{
S. Raymond
}

\author{
CEA, Institut Nanosciences et Cryogénie, Service de Physique Statistique Magnétisme \\ et Supraconductivité, laboratoire Magnétisme et Diffraction Neutronique, \\ 38054 Grenoble Cedex, France
}

\begin{abstract}
Résumé. Ce cours passe en revue les briques fondamentales du magnétisme nécessaires pour comprendre la diffusion magnétique inélastique des neutrons : la répulsion coulombienne, le couplage spin-orbite, le champ cristallin et l'interaction d'échange inter-atomique. Une introduction aux ondes de spins est ensuite donnée pour différents types d'ordre magnétique. Finalement, deux exemples typiques de réponse dynamique paramagnétique en magnétisme localisé $\left(\mathrm{PrOs}_{4} \mathrm{Sb}_{12}\right)$ et itinérant $\left(\mathrm{Sr}_{2} \mathrm{RuO}_{4}\right)$ sont détaillés afin d'illustrer les concepts introduits.
\end{abstract}

\section{LE SYSTÈME PHYSIQUE, LA SONDE ET LE MODÈLE}

La diffusion inélastique des neutrons est une sonde microscopique qui permet l'investigation de l'espace réciproque $(\mathbf{q}, \omega)$ pour un système donné, q étant le vecteur d'onde et $\hbar \omega$ l'énergie. Lors d'une expérience, on mesure le nombre de neutrons diffusés dans une direction donnée et qui ont subit un transfert d'énergie donné, ce qui donne accès à la fonction de diffusion du système $S(\mathbf{q}, \omega)$. La fonction de diffusion est reliée à la partie imaginaire de la susceptibilité dynamique généralisée par le théorème fluctuation-dissipation :

$$
S(\mathbf{q}, \omega)=\frac{1}{1-e^{-\hbar \omega / k_{B} T}} \chi ”(\mathbf{q}, \omega)
$$

En ce qui concerne les excitations magnétiques, la susceptibilité dynamique généralisée $\chi(\mathbf{q}, \omega)$ est la réponse du système à un champ magnétique $\mathbf{H}$ variant en fréquence et en vecteur d'onde. Si $\mathbf{M}$ est l'aimantation associée, on a :

$$
\mathbf{M}(\mathbf{q}, \omega)=\chi(\mathbf{q}, \omega) \mathbf{H}(\mathbf{q}, \omega)
$$

De façon imagée, c'est comme si le neutron créait un champ microscopique variant en fréquence et vecteur d'onde dans l'échantillon et mesurait la réponse du système à ce champ. La susceptibilité dynamique contient toute la physique du système considéré en l'absence du champ $\mathbf{H}(\mathbf{q}, \omega)$, c'est à dire en l'absence de la sonde. C'est une spécificité importante de la diffusion neutronique par rapport à d'autres spectroscopies pour lesquelles il faut prendre en compte l'effet de la sonde sur le système. Dans ce cours nous montrerons des spectres obtenus par diffusion inélastique des neutrons sans aborder ni les techniques expérimentales ni les sections efficaces de diffusion. Tout ce qu'il faut savoir au niveau de ce cours d'introduction est que la position des pics de la fonction de diffusion donne accès aux niveaux d'énergie du système et les intensités sont reliées à des éléments de matrices caractéristiques du système. En reportant la variation éventuelle des énergies caractéristiques $\omega^{*}$ en fonction de $\mathbf{q}$, on obtient ce qui est appelé une relation de dispersion $\omega^{*}(\mathbf{q})$. Il est courant d'utiliser comme unité d'énergie, le meV, le $\mathrm{K}$ ou le $\mathrm{THz}$ avec $1 \mathrm{meV}=11,6 \mathrm{~K}=0,242 \mathrm{THz}$. En ce qui concerne l'espace réciproque, on utilise le

This is an Open Access article distributed under the terms of the Creative Commons Attribution-Noncommercial License 3.0, which permits unrestricted use, distribution, and reproduction in any noncommercial medium, provided the original work is properly cited. 
vecteur de diffusion $\mathbf{Q}$ et le vecteur d'onde $\mathbf{q}$ avec $\mathbf{Q}=\tau+\mathbf{q}$ où $\tau$ est la position d'un centre de zone de Brillouin.

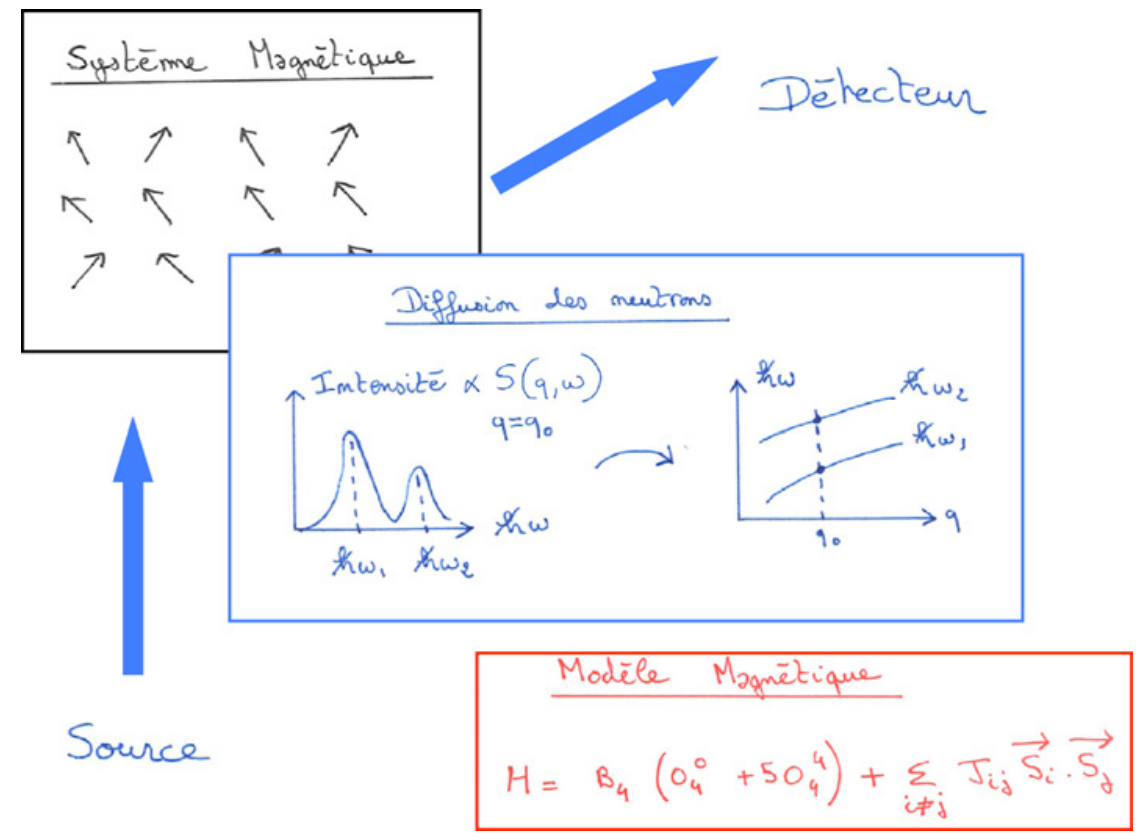

Figure 1. Le système physique, la sonde et le modèle.

L'état fondamental et les niveaux d'énergie du système gouvernent ses propriétés physiques. Certaines grandeurs macroscopiques peuvent être calculées par des méthodes de physique statistique qui prennent en compte la population thermique des différents niveaux. Une autre méthode consiste à exprimer les quantités macroscopiques par rapport à la susceptibilité dynamique. Par exemple, la susceptibilité uniforme mesurée dans un magnétomètre s'écrit :

$$
\chi=\chi^{\prime}(\mathbf{q}=0)=\int \frac{\chi^{\prime \prime}(\mathbf{q}=0, \omega)}{\omega} d \omega
$$

Le temps de relaxation mesuré en Résonance Magnétique Nucléaire s’exprime comme :

$$
\frac{1}{T_{1} T}=\sum_{q, \omega \rightarrow 0}|A(\mathbf{q})|^{2} \frac{\chi ”(\mathbf{q}, \omega)}{\omega}
$$

où $A(\mathbf{q})$ est le coefficient hyperfin. A un degré de sophistication légérement plus élevé et moyennant quelques hypothèses, on peut aussi exprimer la résistivité et la chaleur spécifique dues au fluctuations magnétiques.

Pour juger de la compréhension d'un phénomène physique, il faut comparer les résultats expérimentaux à la description théorique qui est donnée par un modèle, en général associé à un Hamiltonien (et à ses solutions : vecteurs propres, valeurs propres). Cette démarche pour aborder un système physique au moyen de la diffusion des neutrons est schématisée sur la figure 1. Le Hamiltonien magnétique est constitué d'une somme de plusieurs termes qui traduisent la formation d'un moment magnétique à partir de $\mathrm{N}$ électrons, les effets de l'environnement cristallographique sur le moment magnétique ainsi formé et finalement l'interaction entre moments magnétiques :

$$
H=H_{1}+H_{2}+H_{3}+\ldots
$$


Le problème global est extrêmement complexe à résoudre, les termes plus petits seront traités en perturbation des termes beaucoup plus grands. Tout le "jeu" du magnétisme consiste à classer ainsi l'importance des interactions. Le but de ce cours est de passer en revue ces différentes interactions et de les illustrer par des spectres de diffusion inélastique des neutrons.

Le plan du cours est le suivant : La partie 2 traite des briques du magnétisme. Dans la section 2.1, nous rappelons comment se forme un moment magnétique en prenant en compte la répulsion coulombienne et le couplage spin-orbite. Le moment magnétique ainsi formé est placé dans son environnement local et nous traitons dans la section 2.2 du champ cristallin. Ceci conduit à des comportements distincts en particulier pour les deux familles d'importance pour le magnétisme dans la table périodique des éléments : la première série d'éléments de transition $(3 d)$ et la série des terres rares $(4 f)$. Enfin le moment étant placé dans un réseau, nous traitons des interactions entre moments, c'est à dire des interactions d'échange, dans la section 2.3. Le cas du magnétisme itinérant, pour lequel une telle description "pas à pas" du magnétisme n'est pas valide, est ensuite brièvement abordé dans la section 2.4. Les fondements du magnétisme étant posés, nous traitons ensuite des ondes de spin dont l'importance justifie d'y consacrer une partie entière (partie 3). La dernière partie (partie 4) est dédiée à l'introduction de deux exemples typiques de réponse dynamique dans des phases paramagnétiques en magnétisme localisé $\left(\mathrm{PrOs}_{4} \mathrm{Sb}_{12}\right)$ et itinérant $\left(\mathrm{Sr}_{2} \mathrm{RuO}_{4}\right)$.

\section{LES BRIQUES FONDAMENTALES DU MAGNÉTISME}

\subsection{Formation d'un moment magnétique : Répulsion coulombienne et couplage spin-orbite}

Avant de traiter le problème à $\mathrm{N}$ électrons, nous rappelons la notion de moment magnétique pour un électron. Un électron de masse $m_{e}$ et de charge - $e$ en mouvement sur son orbite possède un moment magnétique orbital $\mathbf{M}_{\text {orb }}$ proportionnel à son moment cinétique : $\mathbf{M}_{\mathbf{o r b}}=\frac{-e \hbar}{2 m_{e}} \mathbf{l}$ où $\mathbf{l}$ est l'opérateur moment cinétique orbital. L'unité de moment magnétique est le magneton de $\operatorname{Bohr}\left(1 \mu_{B}=\frac{e \hbar}{2 m_{e}}=9,274 \cdot 10^{-24}\right.$ $\mathrm{Am}^{2}$ ); ainsi $\mathbf{M}_{\mathbf{o r b}}=-\mathbf{l} \mu_{B}$. La seconde contribution au magnétisme provient du spin de l'électron et de l'opérateur spin $\mathbf{s}$ associé avec $\mathbf{M}_{\text {spin }}=-\mathbf{2} \mathbf{s} \mu_{B}$. On donne souvent l'image pour le spin d'un mouvement de rotation de l'électron sur lui-même.

Le Hamiltonien d'interaction pour N électrons autour d'un noyau avec Z protons s'écrit :

$$
H=\sum_{i}\left[\frac{p_{i}^{2}}{2 m_{e}}-\frac{Z e^{2}}{4 \pi \epsilon_{0} r_{i}}\right]+\sum_{i>k} \frac{e^{2}}{4 \pi \epsilon_{0} r_{i k}}+H_{s o}
$$

$p_{i}$ est la quantité de mouvement de l'électron $i$ à la position $r_{i}, r_{i k}$ est la distance entre les électrons $i$ et $k$ et $\epsilon_{0}$ est la permitivité électrique du vide. Le premier terme de l'équation est l'énergie cinétique des électrons, le second terme est l'interaction avec le noyau. Le troisième terme est l'interaction coulombienne entre électrons et le dernier terme est le couplage spin-orbite qui correspond à l'action du champ magnétique créé par le mouvement du noyau dans le référentiel de l'électron. Le traitement de ce problème est extrêmement compliqué et l'approche courante consiste à se ramener à un problème plus simple que l'on sait traiter : celui d'électrons indépendants soumis à un potentiel central qui traduit l'action conjointe du noyau et des autres électrons.

On appelle une configuration un état électronique obtenu à partir du potentiel central : c'est une énumération de $\mathrm{N}$ états à un électron. Chaque état à un électron est caractérisé par 4 nombres quantiques : le nombre principal $n_{i}$, le nombre orbital $l_{i}$ (associé au moment cinétique orbital $\mathbf{l}_{\mathbf{i}}$ avec $0 \leq l_{i} \leq n_{i}-1$ ), le nombre azimutal $m_{i}$ (associé à la projection du moment cinétique avec $-l_{i} \leq m_{i} \leq l_{i}$ ) et le nombre de spin $s_{i}$ associé à la projection du moment de $\operatorname{spin} \mathbf{s}_{\mathbf{i}}\left(s_{i}= \pm 1 / 2\right)$. Rappelons que pour $l_{i}=0,1,2,3$ on utilise les lettres $s, p, d, f$.

La partie non centrale du potentiel lève la dégénerescence de chaque configuration en termes caractérisés par les nombres quantiques $L$ et $S$. Ces nombres caractérisent les états propres et valeurs 
propres de $\mathbf{L}^{\mathbf{2}}$ et respectivement $\mathbf{S}^{\mathbf{2}}$. $\mathbf{L}$ et $\mathbf{S}$ sont les opérateurs de moments cinétique orbital et respectivement de spin totaux obtenus par sommation des moments cinétiques $\mathbf{l}_{\mathbf{i}}$ et de spin $\mathbf{S}_{\mathbf{i}}$ individuels des électrons $\left(\mathbf{L}=\sum_{i} \mathbf{l}_{\mathbf{i}}\right.$ et $\left.\mathbf{S}=\sum_{i} \mathbf{s}_{\mathbf{i}}\right)$. Les deux premières règles de Hund donnent les valeurs de $L$ et $S$ pour le terme fondamental. Ceci correspond à la minimisation de l'interaction coulombienne en tenant compte du principe de Pauli.

Il reste à prendre en compte le couplage spin-orbite. Il s'écrit à l'intérieur de l'espace des $(2 L+1)(2 S+1)$ états:

$$
H_{s o}=\lambda \mathbf{L} . \mathbf{S}
$$

avec $\lambda>0$ ou $<0$ selon le remplissage de la couche. Le couplage spin-orbite augmente avec la charge du noyau et est donc plus important pour les atomes lourds. Il sépare les termes en multiplets caractérisés par le nombre quantique $J$ donné par la troisième règle de Hund. Ce nombre est associé à l'opérateur moment cinétique total $: \mathbf{J}=\mathbf{L}+\mathbf{S}$. Cette approche du couplage des électrons s'appelle le couplage de Russel-Saunders (ou couplage $L-S$ ) et est valable quand l'interaction de spin-orbite est petite devant la répulsion coulombienne. Quand l'interaction spin-orbite est très forte comme par exemple dans les actinides (électrons $5 f$ ), un meilleur point de départ consiste à coupler entre eux chaque $\mathbf{l}_{\mathbf{i}}$ et $\mathbf{S}_{\mathbf{i}}$ afin d'obtenir un $\mathbf{j}_{\mathbf{i}}$ de chacun des électrons puis de coupler ensuite les $\mathbf{j}_{\mathbf{i}}$ entre eux : c'est le coupalge $j-j$.

Si la couche électronique n'est pas complète alors l'atome sera porteur de magnétisme avec un moment magnétique orbital $\left(\mathbf{M}_{\text {orb }}\right)$ et un moment magnétique de $\operatorname{spin}\left(\mathbf{M}_{\text {spin }}\right)$. Pour un atome isolé : $\mathbf{M}_{\text {orb }}=-\mathbf{L} \mu_{B}$ et $\mathbf{M}_{\text {spin }}=-2 \mathbf{S} \mu_{B}$. Dans la suite nous parlerons d'ion magnétique car certains des électrons participant à la liaison chimique, il faut considérer la configuration ionique pour connaître le moment magnétique. Par exemple dans les terres rares, l'état de valence 3+ est courant et correspond à la participation à la liaison chimique de deux électrons $6 s$ et un électron $5 d$ (la couche $4 f$ étant plus interne).

Pour illustrer le paragraphe précédent, prenons comme exemple le praséodyme dont la configuration ionique $\operatorname{Pr}^{3+}$ intervient dans les composés magnétiques. Il y a deux électrons $f$. La première règle de Hund maximise le spin total donnant $S=1$, la seconde règle de Hund maximise $L$ avec $L=5$ et la troisième règle de Hund donne $\mathrm{J}=|\mathrm{L}-\mathrm{S}|$ pour une couche moins que à moitié remplie. Il est courant d'utiliser la notation spectroscopique ${ }^{2 S+1} L_{J}$ soit ${ }^{3} H_{4}$ pour $\operatorname{Pr}^{3+}$. La figure 2 montre une transition électronique mesurée vers $250 \mathrm{meV}$ par diffusion inélastique des neutrons pour le praséodyme [1]. Il s'agit du passage de l'état fondamental caractérisé par $J=4$ à l'état excité caractérisé par $J+1=5$ (configuration ${ }^{3} \mathrm{H}_{5}$ ).

\subsection{Moment magnétique dans son environnement local : Le champ cristallin}

Nous considérons maintenant l'ion magnétique dans son environnement local dans un cristal. Les charges voisines créent un potentiel électrostatique qui est appelé champ cristallin. La théorie du champ cristallin considère les charges comme ponctuelles. Un modèle plus élaboré qui prend en compte la distribution de charge est appelée théorie du champ de ligands. Le potentiel de champ cristallin a une symétrie plus basse que la symétrie sphérique de l'ion libre ce qui entraîne une levée de dégénérescence des niveaux. La figure 3 illustre le cas d'une orbitale $d$ dans un environnement octaédrique avec six charges négatives placées sur les axes $x, y$ et $z$. Les deux orbitales $d$ qui pointent vers ces charges sont défavorisées du point de vue énergétique du fait de la répulsion coulombienne et ont donc une énergie plus grande. Il en résulte une séparation des niveaux entre un triplet fondamental (appelé $t_{2 g}$ ) et un doublet excité (appelé $e_{g}$ ). Il faut aussi ajouter la dégénérescence de spin sur chaque niveau. Pour des charges négatives dans un environnement tétraédrique, le doublet $e_{g}$ a une énergie plus basse. Le potentiel de champ cristallin $V(\mathbf{r})$ s'exprime de façon exacte en harmoniques sphériques. Pour les éléments $d$ (respectivement $f$ ), les règles de sélections entre opérateurs montrent qu'il est nécessaire d'aller jusqu' aux termes d'ordre 4 (respectivement 6). Le théorème de Wigner-Eckart permet d'exprimer 
le potentiel en fonction des composantes d'opérateurs moments cinétiques $\left(L_{x}, L_{y}, L_{z}\right)$ ou moment total $\left(J_{x}, J_{y}, J_{z}\right)$. Ce point est illustré dans le paragraphe suivant.

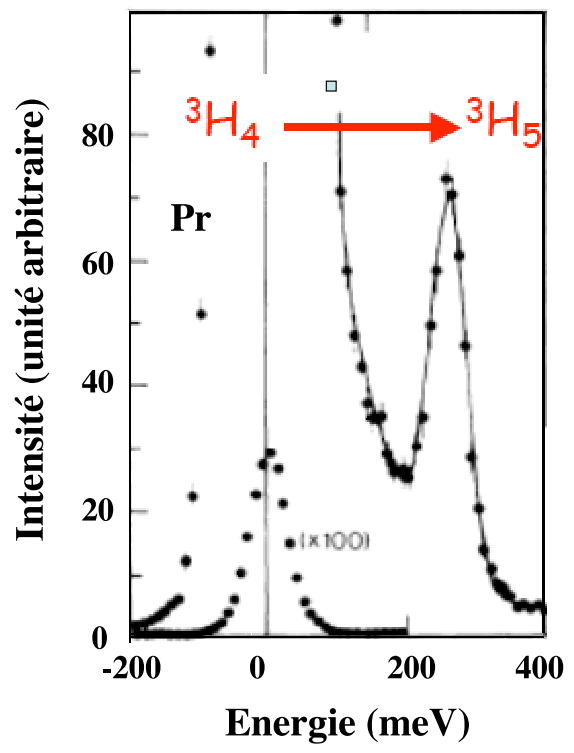

Figure 2. Transition intermultiplet dans le praséodyme [1].

\subsubsection{Les éléments $4 f$}

Ayant introduit le concept de champ cristallin, il faut comparer cette énergie avec la répulsion coulombienne et le couplage spin-orbite. Pour les terres rares, la couche $4 f$ est interne. Les considérations sur l'ion libre s'appliquent : la répulsion coulombienne définit $L$ et $S$, le couplage spinorbite définit $J$ et le champ cristallin opère sur $J$. Prenons par exemple un composé de cérium avec une configuration $\mathrm{Ce}^{3+}$. L'interaction de Coulomb et le couplage spin-obite donnent $J=5 / 2$ comme état fondamental. Le champ cristallin dans un environnement cubique lève la dégénérescence $((2 \mathrm{~J}+1)=6)$ en un doublet $\Gamma_{7}$ et un quartet $\Gamma_{8}$. Le potentiel de champ cristallin s'écrit :

$$
H_{c c}=B_{4}\left(O_{4}^{0}+5 O_{4}^{4}\right)
$$

avec les opérateurs équivalents :

$$
\begin{gathered}
O_{4}^{0}=\left[35 J_{z}^{4}-30 J(J+1) J_{z}^{2}+25 J_{z}^{2}-6 J(J+1)\right] \\
O_{4}^{4}=\frac{1}{2}\left(J_{+}^{4}+J_{-}^{4}\right)
\end{gathered}
$$

Dans le cas particulier du cérium, il n'y a pas de terme d'ordre 6 lorsque l'on considère uniquement le multiplet $J=5 / 2$. Les fonctions d'ondes sont les combinaisons linéaires d'états $\left|J, M_{J}\right\rangle$ suivantes :

$$
\begin{gathered}
\left|\Gamma_{8 B, \pm}\right\rangle=\left|\frac{5}{2}, \pm \frac{1}{2}\right\rangle \\
\left|\Gamma_{8 A, \pm}\right\rangle=\sqrt{\frac{5}{6}}\left|\frac{5}{2}, \pm \frac{5}{2}\right\rangle+\sqrt{\frac{1}{6}}\left|\frac{5}{2}, \mp \frac{3}{2}\right\rangle
\end{gathered}
$$



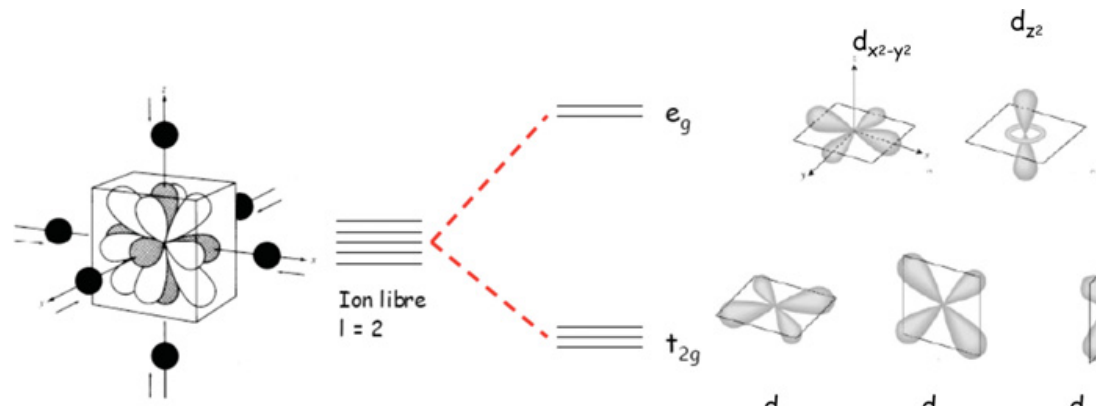

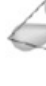

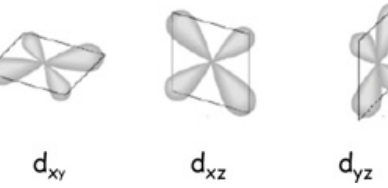

Figure 3. Levée de dégénérescence pour une orbitale $d$ dans un environnement octaédrique.

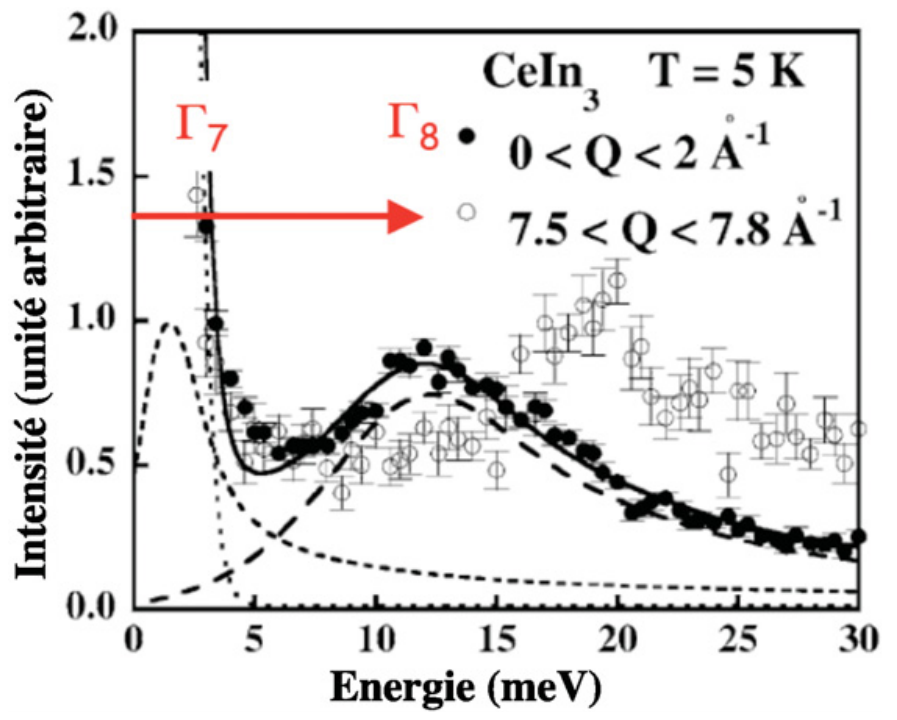

Figure 4. Transition de champ cristallin dans $\mathrm{CeIn}_{3}$ pour $0<\mathrm{Q}<2 \AA^{-1}[2]$.

$$
\left|\Gamma_{7, \pm}\right\rangle=\sqrt{\frac{1}{6}}\left|\frac{5}{2}, \pm \frac{5}{2}\right\rangle-\sqrt{\frac{5}{6}}\left|\frac{5}{2}, \mp \frac{3}{2}\right\rangle
$$

La figure 4 montre la transition de $\Gamma_{7}$ à $\Gamma_{8}$ mesurée vers $12 \mathrm{meV}$ dans le composé cubique $\mathrm{CeIn}_{3}$ [2].

\subsubsection{Les éléments $3 d$}

Pour les éléments $3 d$, l'interaction de champ cristallin est plus grande que le couplage spin-orbite. Le potentiel coulombien définit $L$ et $S$, le champ cristallin agit sur $L$ et le couplage spin-orbite agit sur le niveau de champ cristallin. La troisième règle de Hund n'est pas vérifiée ( $J$ n'est pas un bon nombre quantique). Un cas fréquemment rencontré est celui où il y a une levée de dégénerescence orbitale supplémentaire à celle du champ cristallin par exemple par l'intermédiaire d'une distorsion de réseau. Si l'état fondamental est non-dégénéré, $L$ est nul, il y a disparition du moment orbital ("quenching" en anglais) et on a un magnétisme de spin avec $\mathbf{M}_{\text {spin }}=-\mathrm{gS} \mu_{B}$. Au second ordre le couplage spin-orbite agit en modifiant le facteur de Landé $(g \approx 2 \pm \epsilon)$ et crée une anisotropie magnétique. La figure 5 illustre des transitions de niveaux pour le cluster $\mathrm{Mn}_{12}$ qui réalise un système de spin $S=10$. Le Hamiltonien 
(très simplifié) dont l'origine est le couplage spin-orbite est :

$$
H=-D_{z} S_{z}^{2}
$$

Les niveaux d'énergie sont caractérisés par une valeur propre de $S_{z}(M)$ et les transitions correspondent au passage $M \rightarrow M \pm 1$.

La figure 6 résume la hiérarchie des interactions magnétiques présentées dans les paragraphes précédents pour les électrons $3 d$ et $4 f$. Cette approche n'est pas toujours valable. Dans certains cas pour les éléments $d$, il peut y avoir une compétition entre le champ cristallin et l'énergie de Coulomb lorsque l'on met deux électrons sur la même orbitale. Un cas courant est celui du $\mathrm{Fe}^{2+}\left(3 d^{6}\right)$ dans un environnement octaédrique pour lequel deux états de spin sont possibles. Dans l'état bas spin, les six électrons occupent le niveau fondamental $t_{2 g}$ conduisant à $S=0$. Dans l'état haut spin, deux électrons de "spin up" occupent l'orbitale supérieure $e_{g}$ afin de minimiser la répulsion coulombienne ; on a alors $S=2$.

Pour finir, notons que dans le cas des actinides $(5 f)$, toutes les énergies (Coulomb, spin-orbite, champ cristallin) peuvent être du même ordre de grandeur et le traitement présenté ici par hiérarchie d'interactions n'est plus valide.

\subsection{Moment magnétique dans un réseau : L'échange}

Dans la partie précédente, nous n'avons considéré que les termes locaux $\left(h_{i}\right)$ du Hamiltonien magnétique (Coulomb, spin-orbite et champ cristallin) qui agissent uniquement sur un site magnétique $i$ du réseau cristallin que nous avons appelé ion ou atome magnétique. Nous allons considérer maintenant les interactions $\left(h_{i, j}\right)$ entre les sites $i$ et $j$ et en particulier l'interaction d'échange inter-atomique. Le Hamiltonien magnétique s'écrit alors :

$$
H=\sum_{i} h_{i}+\sum_{i, j} h_{i, j}
$$

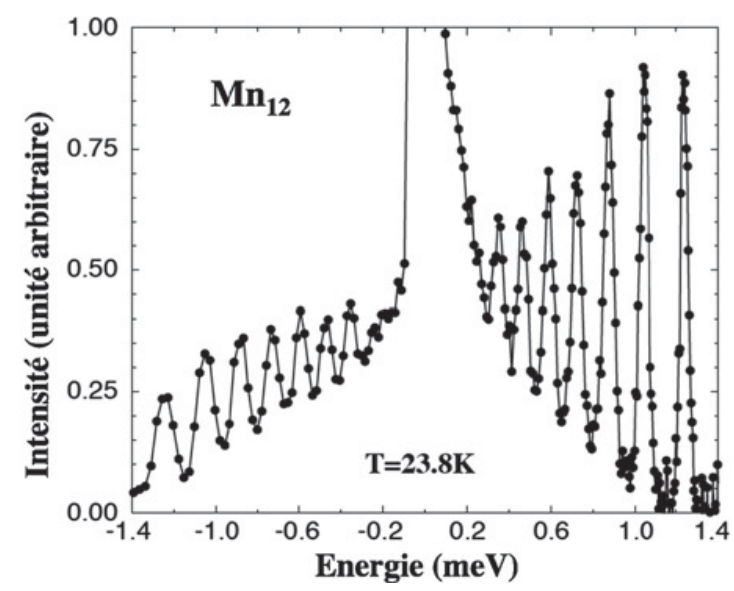

Figure 5. Transitions $M \rightarrow M \pm 1$ de la molécule $\mathrm{Mn}_{12}$ [3].

Dans l'espace récriproque, le premier terme conduit à des phénomènes indépendants du vecteur d'onde $\mathbf{q}$ et le second terme donne une dépendence en $\mathbf{q}$ et donc une dispersion des excitations magnétiques. Dans les exemples montrés précédemment (voir les figures correspondantes 2, 4, 5), les excitations sont non dispersives. La dépendence éventuelle en vecteur de diffusion des spectres mesurés provient de la section efficace des neutrons et non d'une caractéristique intrinsèque du phénomène. 


\section{Eléments $4 f$}

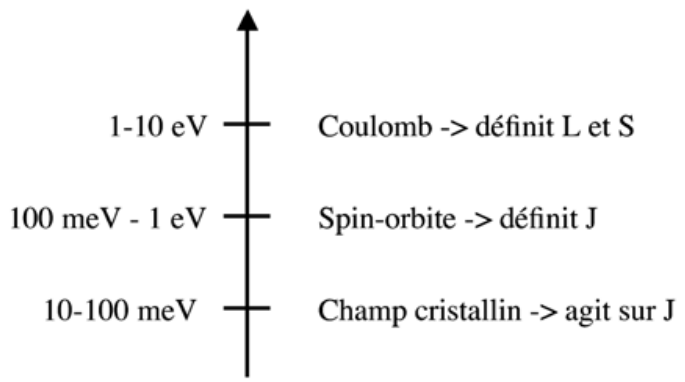

Eléments $3 d$

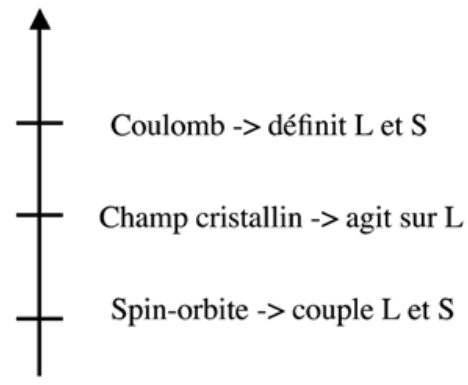

Figure 6. Hiérarchie des interactions magnétiques pour les éléments $4 f$ et $3 d$.

Nous nous intéressons maintenant à l'interaction d'échange : $h_{i, j}=-J_{i, j} \mathbf{S}_{\mathbf{i}} \cdot \mathbf{S}_{\mathbf{j}}$ où $J_{i, j}$ est l'intégrale d'échange entre les sites $i$ et $j$. L'ordre de grandeur de cette intéraction est de 10 à $100 \mathrm{meV}$ pour les éléments $3 d$ et de 1 à $10 \mathrm{meV}$ pour les $4 f$. Il faut alors éventuellement comparer cette échelle d'énergie avec le champ cristallin ou le couplage spin-orbite, c'est à dire situer cette énergie sur la figure 6. Ce point sera évoqué pour la compétition entre champ cristallin et l'interaction d'échange pour les éléments $4 f$ dans la suite du cours. La manifestation expérimentale majeure de l'intéraction d'échange est l'ordre magnétique. En champ moyen, pour un système avec une interaction $J$ uniquement entre $z$ premiers voisins, la température de Curie sous laquelle un ordre ferromagnétique apparaît est $T_{\text {Curie }}=2 z J S(S+1) / 3 k_{B}$.

L'origine de l'interaction d'échange est l'antisymétrie de la fonction d'onde électronique imposée par le principe de Pauli. La fonction d'onde à deux électrons 1 et 2 doit vérifier : $\Psi(1,2)=-\Psi(2,1)$. En l'absence de couplage spin-orbite, on peut factoriser la partie de spin $\chi$ et d'orbite $\phi$ selon les deux possibilités :

$$
\begin{gathered}
\Psi_{I}(1,2)=\phi_{S}(1,2) \chi_{A}(1,2) \\
\Psi_{I I}(1,2)=\phi_{A}(1,2) \chi_{S}(1,2)
\end{gathered}
$$

Les indices $S$ et $A$ dénotent respectivement les fonctions symétriques et antisymétriques. Les deux états $I$ et $I I$ ont une énergie différente si le Hamiltonien contient une interaction (coulombienne) entre électrons. La séparation en énergie entre les deux états est l'énergie d'échange $J_{1,2}=\left(E_{I}-\right.$ $\left.E_{I I}\right) / 2$ et le Hamiltonien effectif s'écrit sous forme d'un "Hamiltonien de spin":

$$
H_{\text {Echange }}=-2 J_{1,2} \mathbf{S}_{\mathbf{1}} \cdot \mathbf{S}_{\mathbf{2}}
$$

Pour $J_{1,2}>0$, l'état fondamental est un triplet de spin total $S=1$ avec trois états $(|\uparrow \uparrow\rangle,|\downarrow \downarrow\rangle$, $|\uparrow \downarrow\rangle+|\downarrow \uparrow\rangle)$. Pour $J_{1,2}<0$, on a un singulet : $S=0$ avec un seul état $(|\uparrow \downarrow\rangle-|\downarrow \uparrow\rangle)$. C'est la répulsion coulombiennne qui est à l'origine de l'échange en produisant une interaction entre électrons mais c'est le principe de Pauli qui en restreignant les fonctions d'ondes possibles fait que le problème électronique se ramène à un problème de spin. La généralisation à $\mathrm{N}$ électrons conduit au Hamiltonien de Heisenberg:

$$
H_{\text {Heisenberg }}=-\sum_{i \neq j} J_{i, j} \mathbf{S}_{\mathbf{i}} \cdot \mathbf{S}_{\mathbf{j}}
$$

Echange intra - atomique : Dans la discussion précédente, les électrons ne sont pas forcément sur un réseau. L'interaction d'échange intra-atomique concerne des électrons d'un même atome. On a en général $J>0$; la fonction d'onde spatiale est antisymétrique ce qui minimise la répulsion coulombienne. Le terme d'échange intra-atomique est en fait contenu dans le Hamiltonien (2.1) mais 
nous ne l'avons pas traité de façon explicite dans le paragraphe 2.1 par souci de pédagogie. En effet les manifestations de l'échange intra-atomique sont entièrement comprises dans la première règle de Hund.

Echange inter-atomique : En ce qui concerne l'échange inter-atomique (entre sites $i$ et $j$ ) qui nous intéresse dans la suite, il faut prendre en compte le fait que l'énergie cinétique des électrons est minimisée par formation de liaison chimique. En général, l'orbitale liante est favorisée $(J<0)$. La figure 7a) montre le lien entre le signe de l'échange et le recouvrement des orbitales pour un échange direct. Dans les solides, l'interaction d'échange directe requiert un recouvrement d'orbitale. Les distances entre ions magnétiques ne permettant pas toujours ce recouvrement, de nombreux mécanismes d'échange sont indirects.

Dans les isolants à base d'élements $d$, le mécanisme de super-échange est très courant lorsque les ions magnétiques sont séparés par des ions non-magnétiques. Il fait intervenir une orbitale $p$ (de l'oxygène par exemple) qui s'hybride avec les orbitales $d$. Le ou les électrons $p$ ainsi délocalisés sur deux ions $d$ produisent l'interaction d'échange indirecte. Le signe de $J$ dépend de l'orbitale, du nombre d'électrons en jeu et de l'angle de la liaison.

Dans les métaux, l'échange indirect de RKKY (Ruderman Kittel Kasuya Yosida) est courant pour des moments localisés sur des sites (en particulier pour les $4 f$ ). L'interaction entre moments magnétiques se fait pas l'intermédiare des électrons de conduction. L'interaction est oscillante avec la distance entre sites et s'écrit $J \propto I^{2} \chi_{\text {conduction }}$ où $I$ est l'intéraction d'échange entre le moment localisé et les électrons de conduction et $\chi_{\text {conduction }}$ est la susceptibilité des électrons de conduction. La figure 7b) schématise les interactions d'échange indirectes de type super-échange et RKKY.

a) échange direct
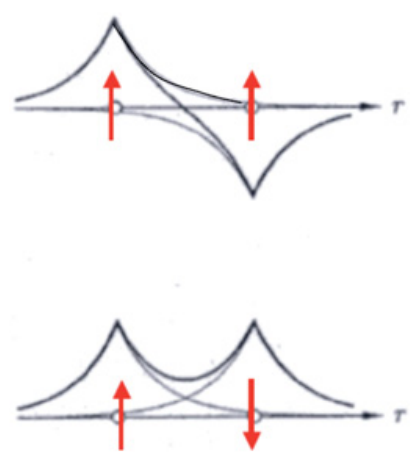

b) échange indirect

Super-échange

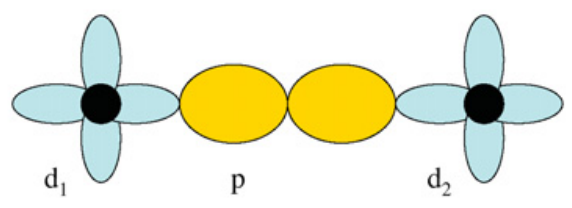

RKKY

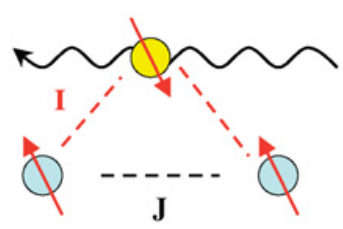

Figure 7. a) Echange direct : en haut la partie spatiale de la fonction d'onde est antisymétrique avec un état de spin triplet; en bas la partie spatiale de la fonction d'onde est symétrique avec un état de spin singulet. b) Echange indirect : en haut super-échange dans un isolant $3 d$ (l'interaction résultante entre électrons $d$ fait intervenir une hybridation entre les électrons $p$ et $d$ ); en bas échange RKKY pour un métal avec des moments localisés ( $I$ est l'interaction entre un moment localisé et les électrons de conduction).

\subsection{Magnétisme de bandes}

Dans les métaux pour lesquels les électrons sont itinérants, la description précédente qui correspond à celle de moments magnétiques localisés sur des sites $i$, n'est pas adaptée. Une description à partir de la structure de bande est plus pertinente. Si on prend en compte le fait que les électrons ont un spin, 
il y a une dissymétrie entre les spins up et down. La façon la plus simple de traiter le problème est due à Stoner. Deux électrons sur la même orbitale ont forcement des spins opposés d'après le principe de Pauli. Ils se repoussent donc plus que des électrons de même spin. Celà revient à introduire une interaction du type $U n_{\uparrow} n_{\downarrow}$. Du fait de cette interaction, une séparation (splitting en anglais) spontanée des bandes $\uparrow$ et $\downarrow$ peut se produire afin de minimiser l'énergie de Coulomb. Ceci se produit si le critère de Stoner est satisfait : $U N\left(E_{F}\right)>1$ où $N\left(E_{F}\right)$ est la densité d'état au niveau de Fermi. On a alors un état ferromagnétique de bande avec comme conséquence remarquable une valeur de moment magnétique qui n'est pas un nombre entier ou demi entier de $\mu_{B}$. L'approche de Stoner ne prend pas en compte les détails de la forme de la surface de Fermi. En particulier il peut y avoir des propriétés d'emboîtement (nesting en anglais) de ces surfaces pour des vecteurs particuliers qui conduisent à des interactions antiferromagnétiques ou incommensurables (voir la section 4.2 avec l'exemple de $\mathrm{Sr}_{2} \mathrm{RuO}_{4}$ ).

La probabilité de saut d'un électron d'un site $i$ à un site $j, t_{i, j}$, et la répulsion $U$ entre électrons de spins opposés sont les ingrédients du modèle de Hubbard très important en magnétisme :

$$
H_{\text {Hubbard }}=-\sum_{i, j} t_{i, j, \sigma} c_{i \sigma}^{\dagger} c_{j, \sigma}+\sum_{i} U n_{i, \uparrow} n_{i, \downarrow}
$$

La limite $t \gg U$ correspond au cas du ferromagnétisme des métaux. Pour $U \gg t$ et pour une bande au plus à moitié remplie, on retrouve le Hamiltonien de Heisenberg (2.14) avec $-J=4 t^{2} / U$. La particularité des excitations magnétiques dans un modèle de magnétisme de bande consiste en l'existence de continuum d'excitation dans l'espace $(\mathbf{q}, \omega)$ appelé continuum de Stoner. Ces modes correspondent à une excitation électron-trou entre les bandes $\uparrow$ et $\downarrow$ avec une énergie :

$$
\hbar \omega_{\mathbf{q}}=\epsilon_{\mathbf{k}+\mathbf{q}, \uparrow}-\epsilon_{\mathbf{k}, \downarrow}+\Delta
$$

où $\Delta$ est la séparation entre les deux bandes due à l'échange et $\epsilon_{\mathbf{k}}$ est l'énergie d'un électron sur une bande. La figure 8 schématise ce continuum d'excitations.

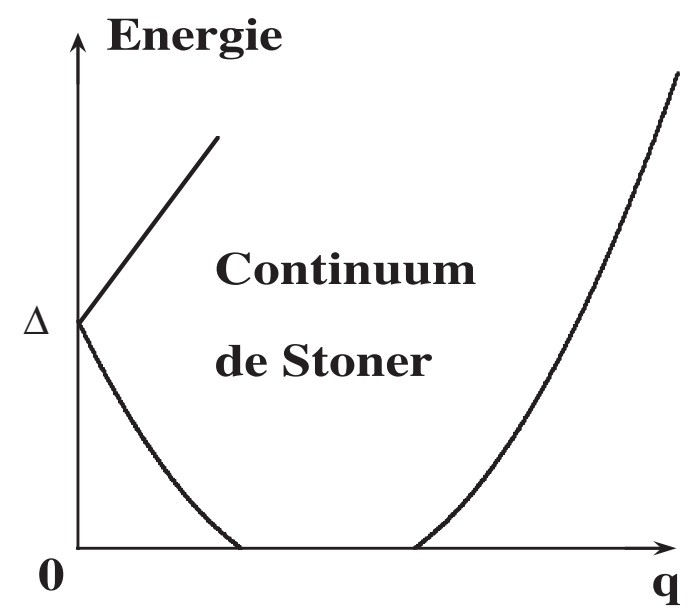

Figure 8. Spectre des excitations de Stoner. Les lignes délimitent le continuum d'excitation dans l'espace $(\mathbf{q}, \omega)$.

\section{LES ONDES DE SPIN}

Dans cette partie nous détaillons les excitations de basses énergies d'un système dont l'état fondamental est ordonné magnétiquement (en opposition à l'état paramagnétique). Il s'agit des ondes de spin auxquelles sont associées un quantum élémentaire : le magnon. La mesure du spectre des ondes de 
spin permet de déterminer les intégrales d'échanges $J_{i, j}$. L'importance de ces excitations justifie d'y consacrer une partie entière. Les ondes de spin peuvent être vues comme l'équivalent en magnétisme des phonons (transverses) pour les excitations structurales (la transition paramagnétique-ordre magnétique correspondant à la transition liquide-solide).

\subsection{Cas ferromagnétique}

Soit $|\Phi\rangle$ l'état fondamental du système pour lequel la projection du moment selon l'axe de quantification $z$ a sa valeur maximale, $S$, sur tous les sites magnétiques. Considérons un état avec le site $j$ ayant une projection de moment selon $z$ diminuée de $S$ à $S-1:|j\rangle=S_{j}^{-}|\Phi\rangle$. Il est aisé de montrer que cet état n'est pas un état propre du Hamiltonien de Heisenberg. Au contraire un état propre est construit comme une combinaison linéaire de tous les états $|j\rangle$ avec $|\mathbf{q}\rangle=\sum_{j} \exp \left(i \mathbf{q} \cdot \mathbf{R}_{j}\right)|j\rangle$. Le site ayant une projection de spin $S-1$ selon $z$ est ainsi délocalisé dans l'espace. On parle d'une onde de spin avec le vecteur d'onde q. On comprend ici l'intérêt de l'espace réciproque puisque un état propre est caractérisé par q. Celà vient du fait qu'une transformée de Fourier diagonalise une forme quadratique. Dans l'espace des q, l'Hamiltonien de Heisenberg s'écrit en effet :

$$
H=-\sum_{\mathbf{q}} J_{-\mathbf{q}} \mathbf{S}_{\mathbf{q}} \cdot \mathbf{S}_{-\mathbf{q}}
$$

avec $J_{-\mathbf{q}}=\sum_{i \neq j} J_{i, j} e^{i \mathbf{q}\left(\mathbf{R}_{\mathbf{i}}-\mathbf{R}_{\mathbf{j}}\right)}$ et $\mathbf{S}_{\mathbf{q}}=\sum_{i} \mathbf{S}_{i} e^{-i \mathbf{q} \cdot \mathbf{R}_{\mathbf{i}}}$. Cette forme étant diagonale, la résolution du problème est facilitée. Cependant les opérateurs $\mathbf{S}_{\mathbf{q}}$ ont des relations de commutation plus complexes que les opérateurs $\mathbf{S}_{\mathbf{i}}$. La résolution du problème s'éffectue en exprimant les opérateurs de spins en opérateurs bosoniques ou fermioniques qui ont des relations de commutations plus simples. On introduit pour les ondes de spin un boson, le magnon (qui correspond au changement $\Delta S=1$ du système). Soit $a_{i}^{\dagger}$ l'opérateur de création d'un magnon au site $i$ et $a_{i}$ l'opérateur d'annihilation. Pour $n_{i}=a_{i}^{\dagger} a_{i}$, le nombre de magnon dans le système au site $i$, on a $S_{i}^{z}=S-n_{i}$. Dans le traitement le plus courant on linéarise la relation entre les opérateurs de spins et la représentation en bosons. On obtient alors un ensemble d'oscillateurs harmoniques indépendants et le Hamiltonien s'écrit :

$$
H=\sum_{\mathbf{q}} \hbar \omega_{\mathbf{q}} a_{\mathbf{q}}^{\dagger} a_{\mathbf{q}}
$$

où $a_{\mathbf{q}}$ est la transformée de Fourier de $a_{i}$. La relation de dispersion associée est :

$$
\hbar \omega_{\mathbf{q}}=2 S\left[J_{0}-J_{\mathbf{q}}\right]
$$

Cette approche des ondes de spins est valable pour les spins "classiques" (c.-à-d. pour $S$ grand). Pour une chaîne de spins avec un échange $J$ ferromagnétique entre plus proches voisins distants de $a$, on a :

$$
\hbar \omega_{q}=4 S J[1-\cos (q a)]
$$

La figure 9a) montre l'image classique d'une onde de spin ferromagnétique qui correspond à la précession collective des moments autour de leur position d'équilibre. La relation de dispersion pour la chaîne ferromagnétique est montrée sur la figure 9b). La figure 10 montre des spectres d'onde de spin mesurés dans le composé ferromagnétique cubique $\operatorname{EuS}\left(T_{\text {Curie }}=16,6 \mathrm{~K}\right)$ qui a une configuration magnétique ${ }^{8} S_{7 / 2}$ avec un moment magnétique de spin uniquement. La dispersion obtenue selon plusieurs directions est également montrée sur la même figure [4]. Cette mesure permet de déterminer les interactions d'échange entre premiers voisins $(0,221 \mathrm{~K})$ et seconds voisins $(-0,1 \mathrm{~K})$. Le signe moins indique que l'interaction entre seconds voisins est antiferromagnétique dans ce composé.

Jusqu'à présent, nous avons considéré le Hamiltonien de Heisenberg isotrope. Une anisotropie courante est du type $-D_{z} S_{z}^{2}$ (voir formule 2.9). Si $D_{z}>0$, le système a une anisotropie axiale, la direction préférentielle des moments est selon $z$. Il y a un gap à $q=0$ dans le spectre d'excitation qui 
a)

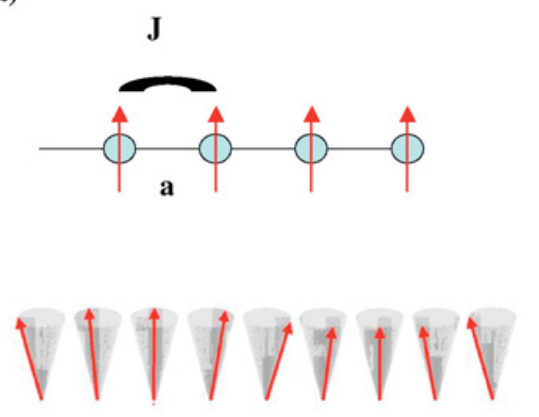

b)

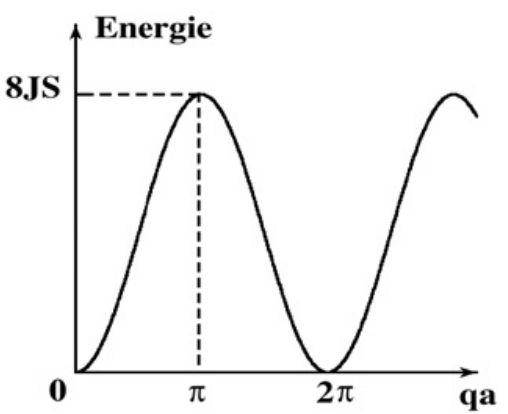

Figure 9. a) Schéma du mouvement de précession de la chaîne de spin ferromagnétique. b) Relation de dispersion associée.
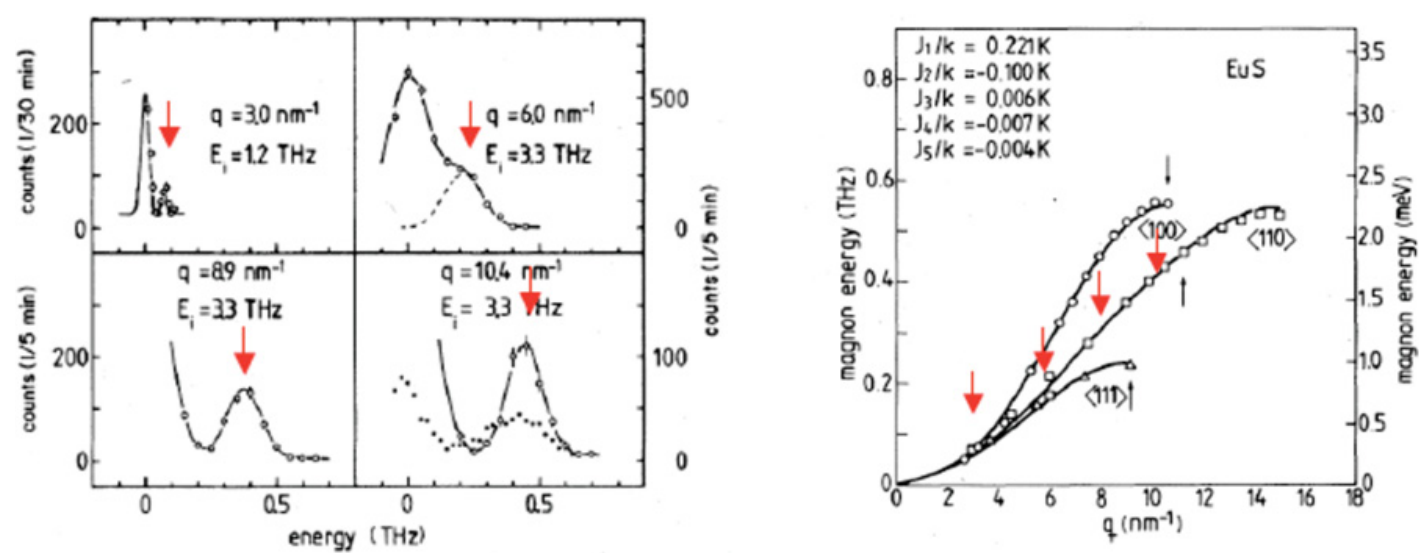

Figure 10. Spectres d'onde de spin et relation de dispersion dans le composé ferromagnétique EuS [4]. Les flèches rouges indiquent les magnons sur les spectres mesurés et leur position est reportée sur la courbe de dispersion.

traduit le fait qu'il faille payer un coût en énergie pour diminuer $S_{z}$. La dispersion devient :

$$
\hbar \omega_{\mathbf{q}}=2 S\left[J_{0}-J_{\mathbf{q}}+D_{z}\right]
$$

Pour un système avec une anisotropie planaire $\left(D_{z}<0\right)$, le traitement est plus complexe, il n'y a pas de gap à $q=0$.

Un autre type d'anisotropie est l'anisotropie de champ cristallin pour les composés de terre rare en particulier. Comme évoqué dans la section 2.2, il faut comparer $H_{c c}$ et $H_{\text {Heisenberg. Pour les terres rares }}$ lourdes (Gd, Tb, Dy...), l'échange est plus fort que le champ cristallin et on peut observer des ondes de spins. Pour les terres rares légères ( $\mathrm{Ce}, \mathrm{Pr})$, le champ cristallin est plus fort et les excitations de plus basse énergie sont des excitons. Il s'agit d'une excitation de champ cristallin qui se propage de site en site par l'échange (voir la section 4.1 avec l'exemple de $\mathrm{PrOs}_{4} \mathrm{Sb}_{12}$ ).

Enfin mentionnons le fait que l'interaction d'échange peut être anisotrope :

$$
H=-\sum_{i, j, \alpha, \beta} J_{i, j}^{\alpha, \beta} S_{i}^{\alpha} S_{i}^{\beta}
$$


et qu'une partie antisymétrique peut exister :

$$
H=\sum_{i, j} \mathbf{D} \cdot\left(\mathbf{S}_{\mathbf{i}} \times \mathbf{S}_{\mathbf{j}}\right)
$$

Cette interaction est connue sous le nom de Dzyaloshinsky-Moriya. Le vecteur D est nul s'il y a un centre d'inversion entre les sites $i$ et $j$.

\subsection{Cas général}

La description d'onde de spin pour une structure magnétique quelconque est assez mal documentée du fait que les choses sont souvent considérées au cas par cas en écrivant des Hamiltoniens particuliers comme pour l'antiferromagnétisme ou le ferrimagnétisme. Le cas général consiste en une structure magnétique quelconque (colinéaire ou non) décrite par un vecteur de propagation $\mathbf{k}$ et avec éventuellement plusieurs atomes magnétiques par maille. Le but est de se ramener au cas ferromagnétique bien connu. La méthode la plus générale consiste ainsi à introduire, sur chaque site magnétique $l$, un repère local dont l'axe $z$ correspond à la direction de la position d'équilibre du spin au point $l$. L'introduction des opérateurs bosoniques se fait dans le repère local comme dans le cas ferromagnétique. Une explication de la méthode est donnée dans le livre de Y. Izyumov et $\mathrm{N}$. Chernoplekov. La forme la plus générale pour une forme quadratique en opérateur de spins est (à comparer avec 3.2) :

$$
\begin{aligned}
H= & \sum_{\mathbf{q}} \sum_{i, j} \sum_{\mu \nu}\left\{B_{i j}\left(\mathbf{k}_{v}, \mathbf{k}_{\mu}, \mathbf{q}\right) \mathrm{b}_{\mathbf{i}}^{\dagger}\left(\mathbf{q}+\mathbf{k}_{\mathbf{l}}\right) \mathrm{b}_{\mathbf{j}}\left(\mathbf{q}-\mathbf{k}_{v}\right)\right. \\
& +A_{i j}\left(\mathbf{k}_{\mu}, \mathbf{k}_{v}, \mathbf{q}\right) \mathbf{b}_{\mathbf{i}}\left(-\mathbf{q}-\mathbf{k}_{\mathbf{l}}\right) \mathrm{b}_{\mathbf{j}}\left(\mathbf{q}-\mathbf{k}_{v}\right) \\
& \left.+A_{i j}^{*}\left(\mathbf{k}_{\mu}, \mathbf{k}_{v}, \mathbf{q}\right) \mathbf{b}_{\mathbf{i}}^{\dagger}\left(-\mathbf{q}-\mathbf{k}_{\mu}\right) \mathrm{b}_{\mathbf{j}}^{\dagger}\left(\mathbf{q}-\mathbf{k}_{v}\right)\right\}
\end{aligned}
$$

Les indices $i$ donnent le nombre d'atomes magnétiques par maille, les vecteurs $\mathbf{k}_{\mu}$ sont les composantes de Fourier permettant de passer du repère cartésien au repère local pour les structures non commensurables. S'il y a $n$ atomes magnétiques par maille, il y a $n$ modes d'ondes de spins. La figure 11 illustre ce point pour la magnétite $\left(\mathrm{Fe}_{3} \mathrm{O}_{4}\right)$ qui est un composé ferrimagnétique avec deux sites magnétiques distincts dans la maille. On observe un mode acoustique et un mode optique d'onde de spin [5].

Nous donnons dans la suite les résultats pour l'antiferromagnétisme à deux sous-réseau et l'hélice simple.

\subsubsection{Cas antiferromagnétique}

Pour un antiferromagnétique à deux sous réseaux, on a deux modes d'onde de spin. Dans le cas le plus simple, les deux modes sont dégénérés. Pour $q=0$, les deux sous réseaux ont des moments qui sont opposés et précessent dans des sens opposés. En bord de zone, les spins d'un des sous réseaux sont immobiles. Ceci est montré sur la figure 12 et est rarement explicité dans la littérature [6]. Un champ magnétique appliqué selon la direction des moments magnétiques lève la dégénerescence entre les deux branches car l'équivalence des deux sous-réseaux n'est plus préservée. Pour une chaîne antiferromagnétique de spins classiques, on a :

$$
\hbar \omega_{q}=4|J \operatorname{Ssin}(q a)|
$$




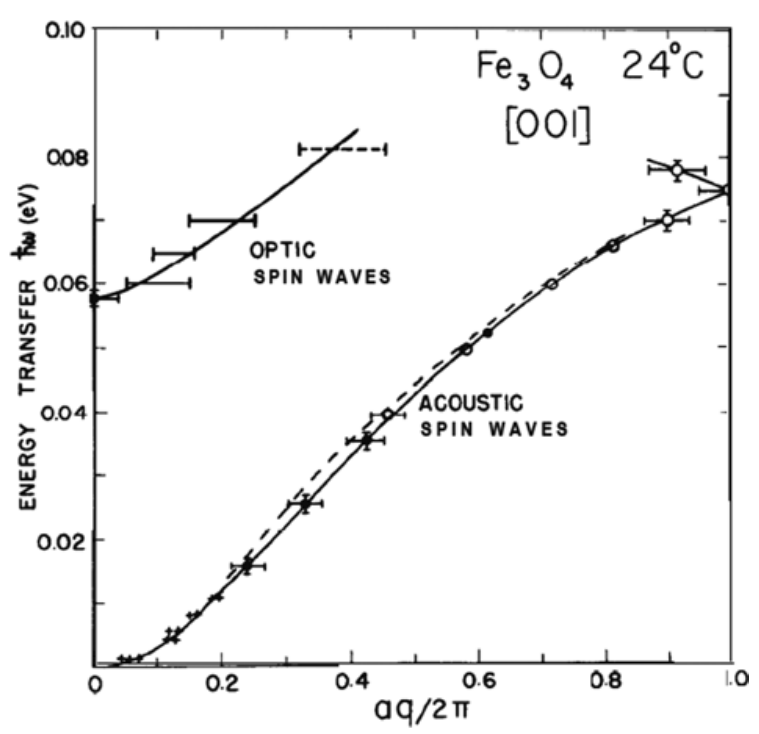

Figure 11. Modes acoustiques et optiques d'onde de spin dans la magnétite [5].

La dispersion est linéraire à petit $q$. En plus de la différence de périodicité avec la chaîne ferromagnétique, on peut aussi noter la valeur deux fois plus petite du maximum d'énergie en bord de zone (4JS au lieu de $8|J| S)$.

\subsubsection{Cas hélimagnétique}

Pour une hélice simple avec les moments dans un plan perpendiculaire au vecteur de propagation $\mathbf{k}$, la relation de dispersion est donnée par :

$$
\left(\hbar \omega_{\mathbf{q}}\right)^{2}=2 S\left[J_{\mathbf{k}}-J_{0}\right]\left[J_{\mathbf{k}}-\frac{1}{2}\left(J_{\mathbf{q}+\mathbf{k}}+J_{\mathbf{q}-\mathbf{k}}\right)\right]
$$

Quand on considère la section efficace de diffusion neutronique pour une telle hélice, il faut prendre en compte le fait que l'on peut observer, pour un $\mathbf{q}$ donné, trois modes pour $\omega_{\mathbf{q}}, \omega_{\mathbf{q}+\mathbf{k}}$ et $\omega_{\mathbf{q}-\mathbf{k}}$. Les vecteurs 0 , $\mathbf{k}$ et -k sont les trois vecteurs qui interviennent dans la description du repère local par rapport au repère cartésien (les vecteur $\mathbf{k}_{\mu}$ de (3.9)). Le fait que l'on puisse mesurer ces trois modes est documenté pour le composé $\mathrm{NiBr}_{2}$ (avec en plus la complexité des domaines magnétiques) [7,8]. La Figure 12 montre la relation de dispersion pour la chaîne quantique frustrée de spin $1 / 2 \mathrm{LiCuVO}_{4}$ ayant un ordre tridimensionnel avec une structure hélimagnétique en dessous de 2,1 K [9]. La dispersion est raide selon $b^{*}$ et plate selon les directions perpendiculaires à $b^{*}$. Bien que nous ayons mentionné que le concept d'onde de spin ne s'applique pas pour les systèmes de spins quantiques, il se trouve que la partie basse du spectre des excitations d'une chaîne Heisenberg de spin 1/2 se décrit bien par une dispersion d'onde de spin classique avec des paramètres renormalisés. Le minimum de la dispersion des excitations dans la phase ordonnée de $\mathrm{LiCuVO}_{4}$ correspond au vecteur de propagation $(0,0.532,0)$; sa proximité de la valeur antiferromagnétique est un effet quantique.

\subsection{Remarques}

Le concept d'onde de spin a été introduit par le Hamiltonien de Heisenberg mais il ne se limite pas à ce modèle. Le mode d'onde de spin est le mode de Goldstone de la transition magnétique, c'est à dire 


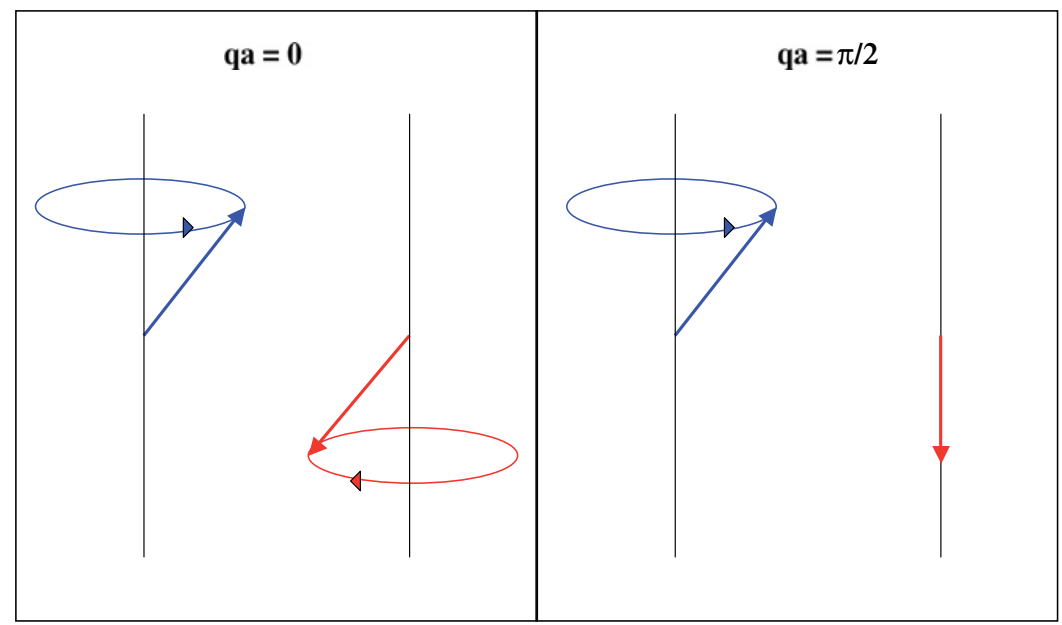

Figure 12. Représentation de la position des spins des deux sous-réseaux pour une onde de spin dans un composé antiferromagnétique en centre $(q a=0)$ et bord de zone $(q a=\pi / 2)$. Les deux modes (souvent dégénérés) correspondent à inverser le rôle des sous-réseaux "bleus" et "rouges".

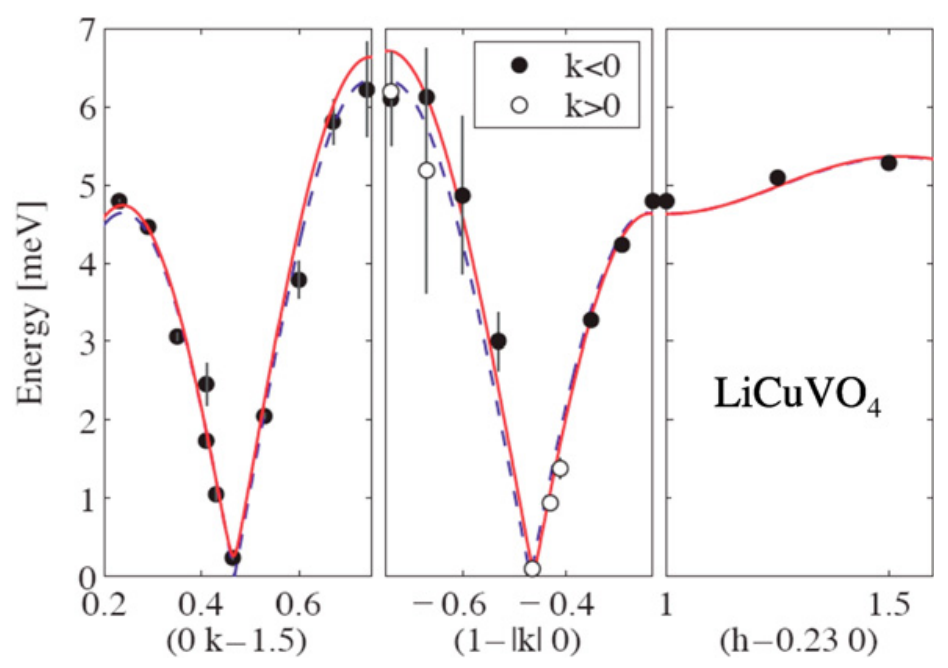

Figure 13. Dispersion des excitations magnétiques dans $\mathrm{LiCuVO}_{4}[9]$.

le mode pour lequel $\omega_{q} \rightarrow 0$ pour $q \rightarrow 0$. Son existence est "garantie" pour tout système magnétique pour lequel la symétrie brisée est continue. C'est le cas bien sûr pour le Hamiltonien de Heisenberg qui est invariant par rotation globale des spins mais ce n'est pas le cas pour un système Ising dont la symétrie brisée est discrète (symétrie up-down). Tout terme qui ne respecte pas l'invariance par rotation (comme certaines anisotropies, voir section 3.1) conduit à une énergie finie pour $q \rightarrow 0$. La description des ondes de spin en terme de mode de Goldstone permet de comprendre pourquoi il y a deux modes dans un antiferromagnétique simple : le mode à $\mathbf{q}=0$ traduit l'invariance du Hamiltonien par rotation et le mode à $\mathbf{q}=\mathbf{k}$ traduit l'invariance par inter-changement des deux sous-réseaux. D'après ces considérations générales, on a donc aussi bien des ondes de spin dans le cas du magnétisme itinérant (section 2.4). Nous ne traitons pas le formalisme associé dans le cadre de ce cours. Le point important est que à grand q, ces excitations bien définies d'onde de spins se "noient" dans le continuum de Stoner. 


\section{EXCITATIONS PARAMAGNÉTIQUES EN MAGNÉTISME LOCALISÉ ET ITINÉRANT}

Cette dernière section vise à développer un peu plus en détail deux types de réponses dynamiques typiques en magnétisme localisé et itinérant à travers deux exemples. Pour ce faire, nous devons au préalable introduire l'approximation la plus courante utilisée pour calculer $\chi(\mathbf{q}, \omega)$ qui est l'approximation de la phase aléatoire (Random Phase Approximation (RPA) en anglais). C'est une approximation de champ moyen pour chaque q et $\omega$. Rappelons que l'approximation du champ moléculaire pour un ferromagnétique consiste à ajouter au champ extérieur appliqué $\mathbf{H}$ un champ interne uniforme proportionnel à l'aimantation $\mathbf{M}$ :

$$
\mathbf{M}=\chi_{0}(\mathbf{H}+I \mathbf{M})
$$

où $\chi_{0}$ est la susceptibilité du système sans interaction. Pour le cas général d'un champ variant en vecteur d'onde et en fréquence :

$$
\mathbf{M}(\mathbf{q}, \omega)=\chi_{0}(\mathbf{q}, \omega)[\mathbf{H}(\mathbf{q}, \omega)+I(\mathbf{q}) \mathbf{M}(\mathbf{q}, \omega)]
$$

On néglige les effets de retard : $I(\mathbf{q})$ est indépendant de la fréquence. D'après la définition (1.2), on a alors l'expression de la susceptibilité :

$$
\chi(\mathbf{q}, \omega)=\frac{\chi_{0}(\mathbf{q}, \omega)}{1-I(\mathbf{q}) \chi_{0}(\mathbf{q}, \omega)}
$$

La susceptibilité sans interaction est donc renforcée par le terme 1/[1-I(q) $\left.\chi_{0}(\mathbf{q}, \omega)\right]$.

\subsection{Exemple en magnétisme localisé : exciton dans $\mathrm{PrOs}_{4} \mathrm{Sb}_{12}$}

$\mathrm{PrOs}_{4} \mathrm{Sb}_{12}$ est le premier supraconducteur non-conventionnel à base de Pr découvert en 2002 [10]. Il a attiré l'attention des expérimentateurs et théoriciens car le rôle des fluctuations magnétiques dans la formation de la supraconductivité est mis en avant. Sans parler de supraconductivité, c'est un exemple très pédagogique pour décrire les excitations magnétiques localisées dans un composé de la famille des terres rares. Le composé $\mathrm{PrOs}_{4} \mathrm{Sb}_{12}$ fait partie de la famille des skutterudites remplies qui cristallise dans le groupe d'espace cubique $\operatorname{Im} \overline{3}$. L'ion $\operatorname{Pr}^{3+}$ se trouve en symétrie tétraédrique $T_{h}$ et le splitting de champ cristallin donne un singulet, deux triplets et un doublet. Nous nous intéressons à la dynamique de spin entre l'état fondamental, le singulet $\Gamma_{1}$ et le premier état excité le triplet $\Gamma_{4}^{2}$. L'écart énergétique entre ces deux niveaux est $\Delta$. La susceptibilité locale pour cette transition de champ cristallin est donnée par:

$$
\chi_{0}(\omega)=\left(f_{0}-f_{1}\right) \frac{\Delta M^{2}}{\Delta^{2}-(\omega+i \epsilon)^{2}}
$$

où $f_{0}$ est la population thermique du fondamental et $f_{1}$, celle de l'état excité. $M$ est l'élément de matrice dipolaire entre les deux niveaux: $M=\left\langle\Gamma_{4}^{2}\left|J_{x}\right| \Gamma_{1}\right\rangle$ et $\epsilon \rightarrow 0$. Cette susceptibilité est indépendante de $\mathbf{q}$ car elle correspond dans l'espace réel à une excitation locale sur un site donné de $\operatorname{Pr}^{3+}$. L'interaction $I(\mathbf{q})$ entre sites est prise en compte par la formule (4.3) et on a alors :

$$
\chi(\mathbf{q}, \omega)=\frac{\left(f_{0}-f_{1}\right) \Delta M^{2}}{\Delta^{2}-(\omega+i \epsilon)^{2}-I(\mathbf{q})\left(f_{0}-f_{1}\right) \Delta M^{2}}
$$

La relation de dispersion est donnée par le pôle de (4.5):

$$
\hbar \omega_{\mathbf{q}}=\sqrt{\Delta\left(\Delta+\left(f_{0}-f_{1}\right) M^{2} I(\mathbf{q})\right)}
$$

Il est important de noter que l'on a affaire à une excitation magnétique dispersive dans une phase paramagnétique. Cette excitation n'a rien à voir avec une onde de spin. L'intéraction $I(\mathbf{q})$ revient ici à propager l'excitation de champ cristallin d'un site à un autre. Ceci est schématisé sur la Figure 14a. 
a)

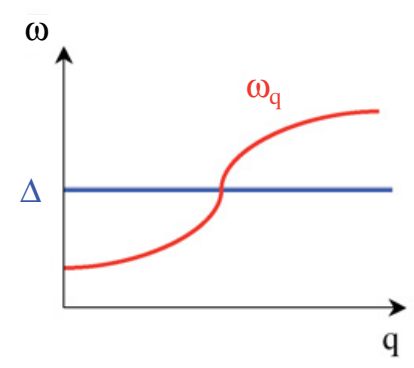

b)

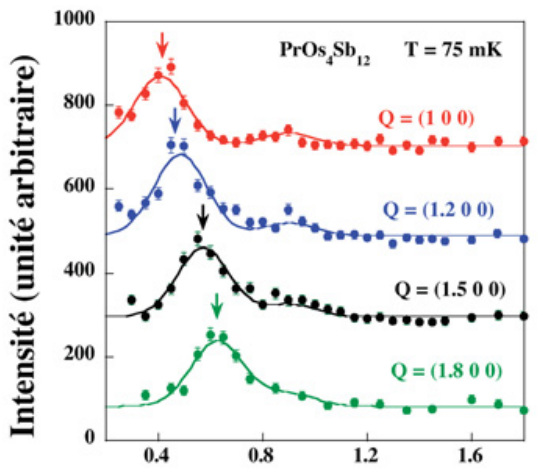

c)

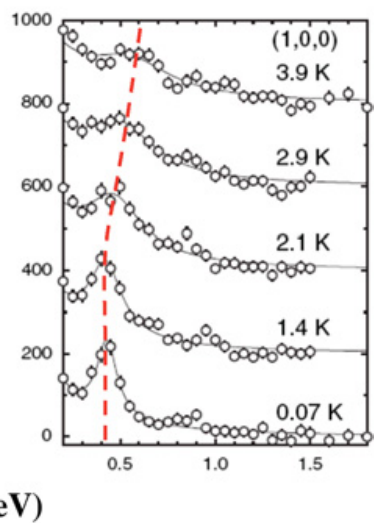

Figure 14. a) Excitation de champ cristallin sans interaction (bleu) et avec interaction entre sites (rouge). b) Dispersion mesurée à $75 \mathrm{mK}$ dans $\mathrm{PrOs}_{4} \mathrm{Sb}_{12}$ selon l'axe $a$. c) Dépendence en température de la transition en $\mathbf{Q}=(1,0,0)[11]$.

Dans le jargon de la physique des terres rares, on appelle ce type d'excitation un exciton. Dans le cas de $\mathrm{PrOs}_{4} \mathrm{Sb}_{12}$, l'interaction n'est pas magnétique de type RKKY mais quadrupolaire [11]. Ici l'interation est maximale au bord de zone de Brilllouin pour $\mathbf{Q}=(1,0,0)$. La dispersion de l'excitation est montrée sur la Figure $14 \mathrm{~b}$ à très basse température $(75 \mathrm{mK})$. On a alors $T \ll \Delta$ et donc $f_{0}=1$ et $f_{1}=0$. Par contre quand on chauffe le système, le niveau excité se peuple, $\left(f_{0}-f_{1}\right)$ diminue, et :

- l'amplitude de la dispersion diminue.

- la position en énergie du minimum de la dispersion augmente $(\operatorname{car} I(\mathbf{Q}=(\mathbf{1}, 0,0))<0)$.

- l'intensité du signal disparaît.

La variation du spectre mesuré en $\mathbf{Q}=(1,0,0)$ en fonction de la température est montrée sur la figure $14 \mathrm{c}$ et illustre ces deux derniers points.

\subsection{Exemple en magnétisme itinérant : $\mathrm{Sr}_{2} \mathrm{RuO}_{4}$}

Le composé $\mathrm{Sr}_{2} \mathrm{RuO}_{4}$ est un supraconducteur non-conventionnel qui a attiré l'attention du fait de sa similarité structurale avec les composés supraconducteurs à haute température critique à base de cuivre [12]. Au niveau de ce cours, c'est un excellent exemple de réponse dynamique typique du magnétisme de bande. L'ion $\mathrm{Ru}^{4+}$ est en configuration $4 d^{4}$ avec un spin $S=1$ sur le niveau fondamental $t_{2 g}$. La surface de Fermi est constituée d'une bande $\alpha$ de trous et d'une bande $\beta$ d'électrons provenant des orbitales $d_{y z}$ et $d_{z x}$. La bande $\gamma$ d'électrons provient des orbitales $d_{x y}$. La surface de Fermi montre des propriétés d'emboîtement entre les bandes $\alpha$ et $\beta$ pour le vecteur $\mathbf{Q}_{0}=(1 / 3,1 / 3,0)$. C'est à dire que $\epsilon_{\alpha}(\mathbf{k}) \approx \epsilon_{\beta}\left(\mathbf{k}+\mathbf{Q}_{\mathbf{0}}\right)$ où $\epsilon$ est l'énergie des électrons. Ceci est illustré sur la figure 15. La susceptibilité sans interaction est donnée par la fonction de Lindhard et correspond aux excitations électron-trou sur la surface de Fermi :

$$
\chi_{0}(\mathbf{q}, \omega)=-2 \mu_{B}^{2} \sum_{\mathbf{k}} \frac{f_{\mathbf{k}+\mathbf{q}}-f_{\mathbf{k}}}{\epsilon_{\mathbf{k}+\mathbf{q}}-\epsilon_{\mathbf{k}}-\hbar \omega+i \epsilon}
$$

où $f_{\mathbf{k}}$ est la distribution de Fermi-Dirac. Cette fonction est grande pour le vecteur de nesting $\mathbf{Q}_{\mathbf{0}}$. Il faut ensuite considérer les interactions entre électrons. D'après les calculs de bande, cette interaction est ferromagnétique est vaut $I(\mathbf{q})=0.43 /\left[1+0.08(a / \pi)^{2} \mathbf{q}^{2}\right]$ [13]. La susceptibilité totale est donnée 


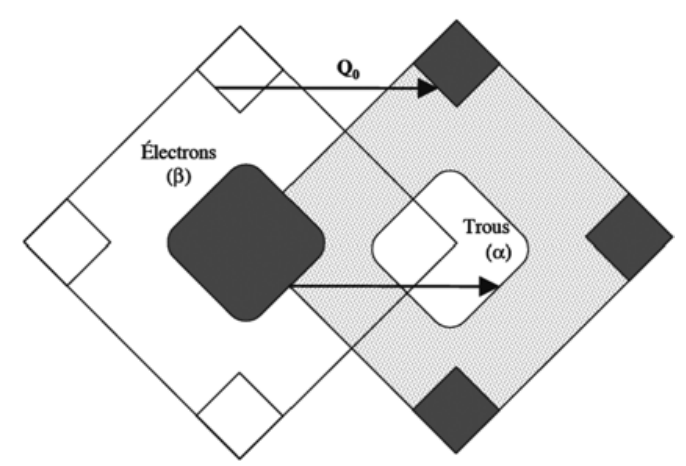

Figure 15. Propriété d'emboîtement des surfaces de Fermi entre les bandes $\alpha$ et $\beta$ de $\mathrm{Sr}_{2} \mathrm{RuO}_{4}$.

a)

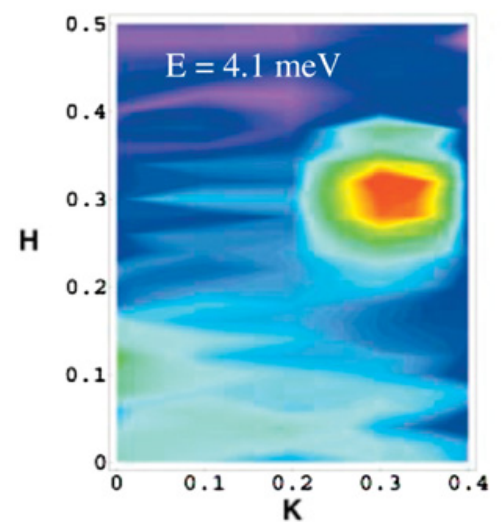

b)

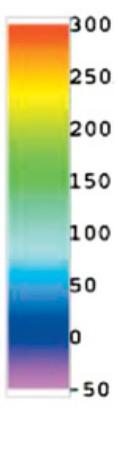

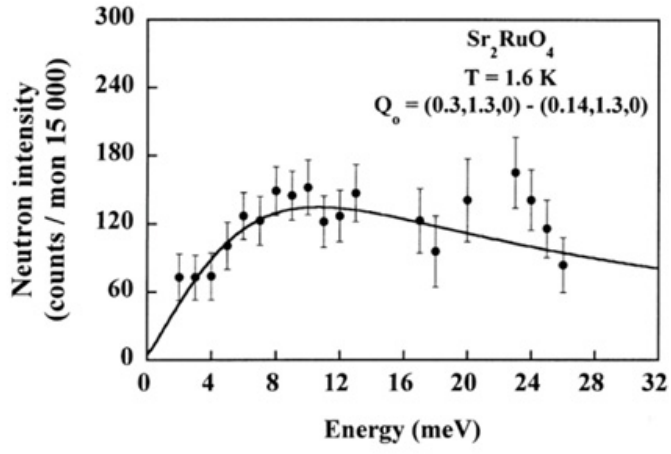

Figure 16. a) Carte d'intensité des excitations magnétiques dans $\mathrm{Sr}_{2} \mathrm{RuO}_{4}$ à $T=10 \mathrm{~K}$ pour une énergie de transfert de $4.1 \mathrm{meV}$ [14]. b) Spectre en énergie des fluctuations incommensurables en $\mathbf{Q}_{\mathbf{0}}$ [15].

par (4.3) et présente deux instabilités : incommensurable par la susceptibilité sans interaction $\chi_{0}(\mathbf{q}, \omega)$ et ferromagnétique par l'interaction $I(\mathbf{q})$. La figure 16a montre une carte d'intensité de diffusion neutronique dans l'espace $(H, K)$ mesurée pour un transfert d'énergie de $4.1 \mathrm{meV}$ à basse température [14]. On observe deux régions de plus forte intensité correpondant aux deux instabilités magnétiques évoquées pour $\mathbf{Q}=0$ et $\mathbf{Q}=\mathbf{Q}_{\mathbf{0}}$. La Figure $16 \mathrm{~b}$ montre un spectre en énergie mesuré pour un vecteur équivalent à $\mathbf{Q}_{\mathbf{0}}$ [15]. La réponse dynamique est diffuse en énergie : il n'y a pas une excitation piquée à une énergie bien définie mais il y a au contraire un continuum d'excitations électroniques caractéristiques du magnétisme itinérant.

\section{CONCLUSION}

Dans ce cours d'introduction, nous avons passé en revue les briques fondamentales du magnétisme : répulsion coulombienne, couplage spin-orbite, champ cristallin et interaction d'échange inter-atomique. Ces concepts ont été illustrés brièvement sur le cas de deux exemples, choisis dans le cadre du magnétisme localisé $\left(\mathrm{PrOs}_{4} \mathrm{Sb}_{12}\right)$ et du magnétisme itinérant $\left(\mathrm{Sr}_{2} \mathrm{RuO}_{4}\right)$. Dans chaque cas, nous avons vu que la fonction de diffusion $S(\mathbf{q}, \omega)$ est une observable importante, qui apporte des informations 
microscopiques essentielles sur la physique de ces systèmes. Nous allons voir en détails dans les prochains cours, comment la section efficace de diffusion inélastique des neutrons permet effectivement de mesurer cette observable. Nous reviendrons également sur le formalisme des ondes de spin, qui constitue un des points importants dans l'étude du magnétisme.

\section{Remerciements}

Je remercie B. Fåk, J.P. Sanchez et S. Petit pour leur relecture attentive du manuscript.

\section{Bibliographie}

\section{Ouvrages généraux de Magnétisme}

Magnétisme, Fondements, Presses Universitaires de Grenoble (1999) Chap. 7-9.

Magnetism in condensed matter, S. Blundell, Oxford University Press (2001).

Magnétisme et supraconductivité, L.P. Levy, CNRS éditions (1997).

Quantum Theory of Magnetism, R.M. White, Springer (1970).

\section{Revue et ouvrage sur les excitations magnétiques}

Magnetic Excitations, W.G. Stirling et K.A. McEwen, Methods of Experimental Physics Vol. 23 Part C Academic Press (1987).

Y.A. Izyumov et N.A. Chernoplekov, Neutron Spectroscopy, Plenum (1994).

\section{Références}

[1] A.D. Taylor et al., Phys. Rev. Lett. 61 (1988) 1309.

[2] W. Knafo et al., J. Phys. Condensed Matter 15 (2003) 3741.

[3] I. Mirebeau et al., Phys. Rev. Lett. 83 (1999) 628.

[4] H.G. Bohn et al., Phys. Rev. B 22 (1980) 5447.

[5] H. Watanabe and B.N. Brokhouse, Physics Letters 1 (1962) 189.

[6] K.-H. Hellwege, Einführung in die Ferstkörperphysik, Springer Verlag 1981.

[7] L. P. Regnault et al., J. Physique 43 (1982) 1283.

[8] P. Day et al., Solid State Comm. 51 (1984) 627.

[9] M. Enderle et al., Europhys. Lett. 70 (2005) 237.

[10] Pour une revue : Y. Aoki et al., J. Phys. Soc. Japan 76 (2007) 051006.

[11] K. Kuwahara et al., Phys. Rev. Lett. 95 (2005) 107003.

[12] Pour une revue : A.P. Mackenzie and Y. Maeno, Rev. Mod. Phys. 75 (2003) 657.

[13] Y. Sidis et al., Phys. Rev. Lett. 83 (1999) 3320.

[14] M. Braden et al., Phys. Rev. B 66 (2002) 064522.

[15] F. Servant et al., Solid State Comm. 116 (2000) 489. 